\title{
ON SOME SIMPLE GROUPS DEFINED BY C. CHEVALLEY
}

\section{BY \\ RIMHAK REE}

Introduction. For any semi-simple Lie algebra $g$ over the field of complex numbers and an arbitrary field $K, \mathrm{C}$. Chevalley [2] defined a group (S) by a uniform method. The group (f) turns out to be simple when $g$ is simple (except for a few exceptional cases), and yields some new classes of simple groups not contained in the theory of classical groups, if, in particular, $g$ is one of the exceptional simple Lie algebras.

In this paper first we consider the case where $g$ is one of the classical algebras $A_{n}, B_{n}, C_{n}, D_{n}$, and identify the group (B) with classical groups, thus answering a question raised in the last section of [2]. Our results are as follows:

(a) if $\mathfrak{g}$ is of the type $A_{n}$, then $B$ is the special projective group $\operatorname{PSL}(n+1, K)$;

(b) if $\mathrm{g}$ is of the type $C_{n}$, then $(5)$ is the quotient group of the symplectic group $\operatorname{Sp}(2 n, K)$ over its center;

(c) if $\mathfrak{g}$ is of the type $D_{n}$, then $(s)$ is the commutator group of the projective orthogonal group defined by the quadratic form $\sum_{i=1}^{n} \xi_{i} \xi_{-i}(3 \leqq n)$;

(d) if $\mathfrak{g}$ is of the type $B_{n}$ and if $K$ is not of characteristic 2 then (B) is the commutator group of the projective orthogonal group defined by the quadratic form $\sum_{i=0}^{n} \xi_{i} \xi_{-i}(2 \leqq n)$; if $\mathfrak{g}$ is of type $B_{n}$ and if $K$ is of characteristic 2 then (B) is a subgroup of $\mathrm{Sp}(2 n, K)$; if, moreover, $K$ is perfect( $\left.{ }^{1}\right)$ then $G=\operatorname{Sp}(2 n, K)$. The author has been unable to identify (f) in case $K$ is not perfect and of characteristic 2 .

Secondly, we consider the case where $g$ is the exceptional algebra $G_{2}$, and identify the group $\&$ with the groups defined by L. E. Dickson $[4 ; 5]$.

1. The group defined by Chevalley. Let $g$ be a semi-simple Lie algebra over the field of complex numbers and $\mathfrak{h}$ a Cartan subalgebra of $\mathfrak{g}$. Denote by $(X, Y)$ the Killing form of $\mathfrak{g}$. For a $\operatorname{root}\left({ }^{2}\right) r$ of $\mathfrak{g}$, we set $H_{r}=2(r, r)^{-1} r$, which is called the co-weight (co-poids) attached to $r$. It is known that the additive group $\mathcal{H}$ generated by all co-weights $H_{r}$ is a free abelian group of rank $n$, where $n$ is the dimension of $\mathfrak{h}$. Chevalley proves the existence of a system $\left\{X_{r}\right\}$ of root vectors satisfying the following conditions (1.1)-(1.2):

$$
\left[X_{r}, X_{-r}\right]=H_{r}
$$

for all roots $r$;

Received by the editors March 18, 1956, and, in revised form, April 12, 1956.

(1) A field $K$ of characteristic $p>0$ is called perfect if every element in $K$ is a pth power of an element in $K$.

(2) Roots of a semi-simple algebra over the field of complex numbers can be identified with elements in the Cartan subalgebra by which the roots are defined. See [7]. 
(1.2) if $r, s$, and $r+s$ are roots then $\left[X_{r}, X_{8}\right]=N_{r, s} X_{r+s}$ with $N_{r, s}= \pm(p+1)$, where $p$ is the greatest integer $i \geqq 0$ such that $s-i r$ is a root.

If a system $\left\{X_{r}\right\}$ of root vectors satisfies (1.1) and (1.3) below then it it also satisfies (1.2) (see [2, pp. 22-23]).

$$
N_{-r,-s}=N_{r, s} \text { for any two roots } r, s \text { such that } r+s \text { is a root. }
$$

Let now $\left\{\tilde{H}_{1}, \cdots, \tilde{H}_{n}\right\}$ be a basis of the group $\mathcal{H}$ and $\left\{X_{r}\right\}$ a system of root vectors satisfying (1.1)-(1.2). Then the set $\left\{\tilde{H}_{1}, \cdots, \widetilde{H}_{n}\right\}$ together with $\left\{X_{r}\right\}$ forms a basis $\left\{\widetilde{H}_{i}, X_{r}\right\}$ of $g$. It is easily seen that the coefficients appearing in the multiplication table of the basis $\left\{\widetilde{H}_{i}, X_{r}\right\}$ are all integers. Chevalley proves that the coefficients of the matrix $A_{r}(t)$ representing the automorphism

$$
x_{r}(t)=\exp t\left(\operatorname{ad} X_{r}\right),
$$

where $t$ is a complex variable, of $\mathfrak{g}$ with respect to the basis $\left\{\tilde{H}_{i}, X_{r}\right\}$ are all polynomials in $t$ with integral coefficients. Therefore for any field $K$ we can define a Lie algebra $g_{K}$ over $K$ by using the basis $\left\{\tilde{H}_{i}, X_{r}\right\}$, and also automorphisms $x_{r}^{*}(t)$, where $t \in K$, of $g_{K}$ by using the matrix $A_{r}(t)$. Then the group considered by Chevalley is the group $\mathbb{S}(\mathfrak{g} ; K)$ (denoted by the symbol $\mathbb{S}^{\prime}$ ' in [2]) generated by all $x_{r}^{*}(t)$, where $r$ runs over all roots of $g$ while $t$ takes on all elements in $K$. This group was proved to be simple except for a few exceptional cases.

Since every $\widetilde{H}_{i}$ is a linear combination of the co-weights $H_{r}$ with integral coefficients, it follows that an element in $\mathbb{S}(\mathfrak{g} ; K)$ is uniquely determined by its effect on $H_{r}^{*}$ and $X_{r}^{*}$, where $H_{r}^{*}$ and $X_{r}^{*}$ are elements in $\mathfrak{g}_{K}$ corresponding to $H_{r}$ and $X_{r}$ in $\mathfrak{g}$, respectively. In particular an element in $(5)$ is the identity if and only if it leaves every $H_{r}^{*}$ and every $X_{r}^{*}$ invariant.

2. The classical algebras. For the classical algebras $A_{n}, B_{n}, C_{n}, D_{n}$ the co-weights $H_{r}$ and the system $\left\{X_{r}\right\}$ of root vectors satisfying (1.1) and (1.3) can be computed easily (cf. [7]).

The algebra $A_{n}$ is the algebra of all $(n+1) \times(n+1)$ matrices of trace zero. Denote by $E_{i j}$ the $(n+1) \times(n+1)$ matrix whose $(i, j)$-entry is 1 and whose other entries are all zero. Then

$$
\left\{H_{r}\right\}=\left\{E_{i i}-E_{j j} \mid i \neq j\right\}, \quad\left\{X_{r}\right\}=\left\{E_{i j} \mid i \neq j\right\},
$$

and if $H_{r}=E_{i i}-E_{j j}$ then $X_{r}=E_{i j}, X_{-r}=E_{j i}$. The mapping $\phi$ which maps a matrix $T$ to $-T^{\prime}$, where $T^{\prime}$ is the transpose of $T$, is an automorphism of $A_{n}$. We have $\phi\left(E_{i j}\right)=-E_{j i}$, and hence it is easily seen that the system $\left\{X_{r}\right\}$ satisfies the condition (1.3).

The algebra $D_{n}$ is the algebra of all $2 n \times 2 n$ matrices $X$ which satisfy $f_{D}(X \xi, \eta)+f_{D}(\xi, X \eta)=0$ identically, where

$$
f_{D}(\xi, \eta)=\xi_{-n} \eta_{n}+\cdots+\xi_{-1} \eta_{1}+\xi_{1} \eta_{-1}+\cdots+\xi_{n} \eta_{-n} .
$$


Denote by $E_{i j}$ the $2 n \times 2 n$ matrix such that $E_{i j} \xi=\xi^{\prime}$, where $\xi_{i}^{\prime}=\xi_{j}, \xi_{k}^{\prime}=0$ for $k \neq i$, and set

$$
F_{i j}=E_{-i j}-E_{-j i}, \quad H_{i j}=F_{i,-i}+F_{j,-j} .
$$

Then we have

$$
\begin{aligned}
& \left\{H_{r}\right\}=\left\{H_{i j} \mid i<j, i \neq-j\right\}, \\
& \left\{X_{r}\right\}=\left\{F_{i j} \mid i<j, i \neq-j\right\},
\end{aligned}
$$

where if $H_{r}=H_{i j}$ then $X_{r}=F_{i j}, X_{-r}=F_{-j,-i}$. The mapping $\phi(T)=-T^{\prime}$ is also an automorphism of $D_{n}$, and hence the system $\left\{X_{r}\right\}$ satisfies (1.3).

The algebra $B_{n}$ may be regarded as the algebra of all $(2 n+1) \times(2 n+1)$ matrices $X$ satisfying $f_{B}(X \xi, \eta)+f_{B}(\xi, X \eta)=0$ identically, where

$$
f_{B}(\xi, \eta)=2 \xi_{0} \eta_{0}+\sum_{i=1}^{n}\left(\xi_{-i} \eta_{i}+\xi_{i} \eta_{-i}\right) .
$$

It is readily seen that a matrix $X=\left(x_{i j}\right)$ belongs to $B_{n}$ if and only if

$$
x_{-i j}+x_{-j i}=0, \quad x_{-i 0}+2 x_{0 i}=0, \quad x_{00}=0
$$

for all $i \neq 0$ and $j \neq 0$. Define the matrices $E_{i j}$ as in the case of algebra $D_{n}$, and set

$$
\begin{aligned}
& F_{i j}=E_{-i j}-E_{-j i}, \quad F_{i 0}=2 E_{-i 0}-E_{0 i}, \quad F_{0 j}=-F_{j 0}, \\
& H_{i j}=F_{i,-i}+F_{j,-j}, \quad H_{i 0}=2 F_{i,-i}, \quad H_{0 j}=2 F_{j,-j}
\end{aligned}
$$

for $i \neq 0, j \neq 0$. Then we see easily that

$$
\left\{H_{r}\right\}=\left\{H_{i j} \mid i<j, i \neq-j\right\}
$$

is the set of all co-weights (with respect to a certain Cartan subalgebra) and that

$$
\left\{X_{r}\right\}=\left\{F_{i j} \mid i<j, i \neq-j\right\}
$$

is a system of root vectors satisfying (1.1), where, $X_{r}=F_{i j}$ and $X_{-r}=F_{-j,-i}$ if $H_{r}=H_{i j}$. This system also satisfies (1.3). This is seen as follows: Let $P$ $=2 E_{00}+\sum_{i=1}^{n}\left(E_{i i}+E_{-i,-i}\right)$. Then the mapping $\phi(X)=-P^{-1} X^{\prime} P$ is an automorphism of $B_{n}$ such that $\phi\left(F_{i j}\right)=-F_{-j,-i}$, that is, $\phi\left(X_{r}\right)=-X_{-r}$ for all roots $r$, and hence (1.3) follows immediately.

The algebra $C_{n}$ is the algebra of all $2 n \times 2 n$ matrices $X$ which satisfy $f_{C}(X \xi, \eta)+f_{C}(\xi, X \eta)=0$ identically, where

$$
f_{C}(\xi, \eta)=\xi_{-n} \eta_{n}+\cdots+\xi_{-1} \eta_{1}-\xi_{1} \eta_{-1}-\cdots-\xi_{n} \eta_{-n} .
$$

Define $E_{i j}$ as in the case of the algebra $D_{n}$ and set

$$
F_{i j}= \begin{cases}E_{-i j}+\operatorname{sign}(i j) E_{-j i}, & \text { if } i \neq j, \\ E_{-i i} & \text { if } i=j,\end{cases}
$$


where sign $k$ denotes +1 if $k>0$, and -1 if $k<0$, and

$$
H_{i j}= \begin{cases}F_{i,-i}+F_{j,-j}, & \text { if } i \neq j, \\ F_{i,-i} & \text { if } i=j .\end{cases}
$$

Then we have

$$
\begin{aligned}
& \left\{H_{r}\right\}=\left\{H_{i j} \mid i \leqq j, i+j \neq 0\right\}, \\
& \left\{X_{r}\right\}=\left\{F_{i j} \mid i \leqq j, i+j \neq 0\right\},
\end{aligned}
$$

and if $H_{r}=H_{i j}$ then $X_{r}=F_{i j}, X_{-r}=F_{-j,-i}$. The mapping $\phi: X \rightarrow-X^{\prime}$ is an automorphism of $C_{n}$ such that $\phi\left(X_{r}\right)=-X_{-r}$ for all roots. Thus (1.3) is also satisfied by the system $\left\{X_{r}\right\}$.

3 . The group $\bar{S}(\mathfrak{g}, K)$. For any two matrices $X, Y$ with complex coefficients we have the formula

$$
\exp (\operatorname{ad} X) \cdot Y=(\exp X) Y(\exp (-X)),
$$

where $(\operatorname{ad} X) \cdot Y=X Y-Y X$. This can be verified easily if we use the identity

$$
\frac{(\operatorname{ad} X)^{k}}{k !} \cdot Y=\sum_{i+j=k} \frac{X^{i}}{i !} Y \frac{(-X)^{j}}{j !} .
$$

For the matrix algebras $\mathrm{g}=A_{n}, C_{n}, D_{n}$, every root vector $X_{r}=F_{i j}$ (we set $F_{i j}=E_{i j}, i \neq j$, for the algebra $A_{n}$ ) given in the preceding section satisfies $X_{r}^{2}=0$, and hence

$$
\exp \left(t X_{r}\right)=I+t F_{i j}
$$

where $I$ is the unit matrix. For the algebra $\mathfrak{g}=B_{n}$, every root vector $X_{r}=F_{i j}$ such that $i \neq 0, j \neq 0$ satisfies $X_{r}^{2}=0$, and hence we have (3.2) for this $X_{r}$ also. For the root vector $X_{r}=F_{i 0}$, of the algebra $B_{n}$ we have $X_{r}^{2}=-2 E_{-i i}, X_{r}^{3}=0$. Therefore

$$
\exp \left(t X_{r}\right)=I+t\left(2 E_{-i 0}-E_{0 i}\right)-t^{2} E_{-i i},
$$

for $i= \pm 1, \pm 2, \cdots, \pm n$. From (3.2) and (3.3) we see that exp $\left(t X_{r}\right)$ can always be represented as a matrix whose entries are polynomials in $t$ with integral coefficients. Now, the matrix $A_{r}(t)$ representing the automorphism $x_{r}(t)$ of $g$ with respect to the basis $\left\{\tilde{H}_{i}, X_{r}\right\}$ can be obtained from the formula

$$
x_{r}(t) \cdot Y=\left(\exp \left(t X_{r}\right)\right) Y\left(\exp \left(-t X_{r}\right)\right),
$$

$Y \in \mathrm{g}$,

which follows from (3.1), and the expression for $\exp \left(t X_{r}\right)$ given in (3.2)-(3.3).

Let $K$ be an arbitrary field and $g$ one of the algebras $A_{n}, B_{n}, C_{n}, D_{n}$. In the preceding section all co-weights $H_{r}$ and root vectors $X_{r}$ of $g$ are represented by matrices with integral coefficients, and consequently, every element in the basis $\left\{\tilde{H}_{i}, X_{r}\right\}$ is also represented as a matrix with integral coefficients. 
Therefore we see that $\mathfrak{g}_{K}$ is represented in this way as a matrix algebra over $K$. For $t \in K$, define the matrix $\exp \left(t X_{r}^{*}\right)$, by the right hand side of (3.3) if $X_{r}$ is one of the root vectors $F_{i 0}, F_{0 j}$ (in case $g=B_{n}$ ), and by that of (3.2) if $X_{r}$ is not, and consider the automorphism $x_{r}^{*}(t)$ defined by $A_{r}(t)$. Then (3.4) yields

$$
x_{r}^{*}(t) \cdot Y^{*}=\left(\exp \left(t X_{r}^{*}\right)\right) Y^{*}\left(\exp \left(-t X_{r}^{*}\right)\right)
$$

for any $Y^{*}$ in $\mathfrak{g}_{K}$. Denote by $\overline{\mathfrak{G}}(\mathfrak{g}, K)$ the multiplicative group of matrices generated by all $\exp \left(t X_{r}^{*}\right)$, where $r$ runs over all roots of $\mathfrak{g}$ while $t$ runs over all elements in $K$. For any $S \in \bar{ङ}$ define the mapping $S^{\sigma}$ of $\mathfrak{g}_{K}$ into itself by $S^{\sigma} \cdot Y^{*}=S Y^{*} S^{-1}$, where $Y^{*} \in g_{K}$. Then (3.5) shows that $S^{\sigma}$ is a product of certain automorphisms of $g_{K}$ of the form $x_{r}^{*}(t)$, and hence belongs to the group $\mathfrak{S}(\mathfrak{g}, K)$ defined in $\S 1$. The mapping $\sigma: S \rightarrow S^{\sigma}$ is clearly a homomorphism of $\overline{(S)}$ onto (5). Denote by 3 the kernel of the homomorphism $\sigma$. By the remark given at the end of $\S 1$, we see that an element $S$ in $\bar{S}$ belongs to $B$ if and only if $S$ commutes with all $X_{r}^{*}$ and $H_{r}^{*}$. If $S \in \mathbb{B}$ commutes with all $X_{r}^{*}$ then $S$ belongs to the center of $\bar{S}$, for $\overline{(S)}$ is generated by elements of the form $\exp \left(t X_{r}^{*}\right)$. Conversely, suppose that $S$ belongs to the center of $\bar{\xi}$. Then $S$ commutes with all $\exp \left(t X_{r}^{*}\right)$. If $\mathrm{g}$ is one of the algebras $A_{n}, C_{n}, D_{n}$, then $\exp \left(t X_{r}^{*}\right)=I+t X_{r}^{*}$. Hence $S$ commutes with all $X_{r}^{*}$. Since $\left[X_{r}^{*}, X_{-r}^{*}\right]=H_{r}^{*}$, we see that $S$ also commutes with all $H_{r}^{*}$. Therefore $S$ belongs to 3 . Thus we have proved that 3 coincides with the center of $\overline{\mathfrak{G}}(\mathfrak{g} ; K)$ if $\mathfrak{g}$ is one of the algebras $A_{n}, C_{n}, D_{n}$. Consider now the case $\mathfrak{g}=B_{n}$. If $S$ belongs to the center of $\bar{B}\left(B_{n}, K\right)$ then $S$ commutes with $\exp X_{r}^{*}$, and hence with $\left(\exp X_{r}^{*}-I\right)^{2}=\left(X_{r}^{*}\right)^{2}$. Therefore, if $K$ is of characteristic $\neq 2$, then $S$ commutes with all $X_{r}^{*}$ and hence belongs to 3 , as before. If $K$ is of characteristic 2 , then from (3.3) we see that $S$ commutes with all $E_{0 i}+E_{-i i}, i= \pm 1, \pm 2, \cdots, \pm n$. Then it follows easily that $S$ is of the form $a I$, with $a \in K$. Therefore $S$ commutes with all $X_{r}^{*}$ and $H_{r}^{*}$, and consequently it belongs to 3 . Thus we have proved

$$
\mathfrak{S}(\mathfrak{g}, K) \cong \bar{\Xi}(\mathfrak{g}, K) / \mathfrak{B},
$$

where $\mathbb{Z}$ is the center of $\overline{\mathscr{S}}(\mathfrak{g}, K)$.

4. The group $\&\left(A_{n}, K\right)$. It is well known that the matrices of the form $I+t E_{i j}$, where $t \in K, i \neq j$, generate the special linear group $\operatorname{SL}(n+1, K)$. Therefore $\bar{\Xi}\left(A_{n}, K\right)=\operatorname{SL}(n+1, K)$. By (3.6) it follows that $\mathcal{B}\left(A_{n}, K\right)$ is isomorphic to the special projective group $\operatorname{PSL}(n+1, K)$.

5. The group $B\left(C_{n}, K\right)$. We show that the group $\bar{\Im}\left(C_{n}, K\right)$ coincides with the symplectic group $\operatorname{Sp}(2 n, K)$ (in its matric representation). It is known [6] that the group $\operatorname{Sp}(2 n, K)$ is generated by the matrices

$$
I+t E_{-i i}, I+t\left(E_{-i j}-E_{-j i}\right),
$$

and

$$
S_{i}=I-E_{i i}-E_{-i,-i}+E_{-i i}-E_{i,-i}
$$


where $t \in K, i, j=1,2, \cdots, n$, except when $n=1$ and $K$ is of order 2 or 3 . In view of $(2.5)-(2.6)$, the first two sets of matrices belong to $\overline{\mathscr{S}}\left(C_{n}, K\right)$. In order to obtain the equality $\overline{B S}=\operatorname{Sp}(2 n, K)$, it thus suffices to show that the matrices $S_{i}$ also belong to $\overline{\mathbb{S}}$. As is mentioned in the preceding section, however, the group $\operatorname{SL}(2, K)$ is generated by matrices of the form $I+t E_{i j}$, where $t \in K, i \neq j$. Therefore $S_{i}$ can be written as a product of matrices of the forms $I+t E_{-i i}$ and $I+t E_{i,-i}$, which belong to $\bar{S}$ by definition. Except when $n=1$ and $K$ is of order 2 or 3 , therefore we obtain $\bar{\xi}=\operatorname{Sp}(2 n, K)$. Hence, by (3.6), $\$\left(C_{n}, K\right)$ is isomorphic to the quotient of the symplectic group $\operatorname{Sp}(2 n, K)$ over its center. It should be noted that $\operatorname{Sp}(2 n, K)$ is the group of all $2 n \times 2 n$ matrices $X$ which satisfy

$$
f_{C}(X \xi, X \eta)=f_{C}(\xi, \eta) \text { identically. }
$$

6. The group $\mathbb{S}\left(D_{n}, K\right)$. First we shall show that the group $\overline{\mathbb{S}}\left(D_{n}, K\right)$ contains the commutator subgroup $\mathfrak{D}^{\prime}$ of the orthogonal group $\mathfrak{D}$ consisting of all $2 n \times 2 n$ matrices which leave the quadratic form $\sum_{i=1}^{n} \xi_{i} \xi_{-i}$ invariant. By (2.2), the group $\overline{(S}$ is generated by the matrices

$$
W_{i, j, t}=I+t\left(E_{i,-j}-E_{j,-i}\right),
$$

where $t \in K$ and $i, j= \pm 1, \cdots, \pm n ; i \neq \pm j$. Denote by $P_{i j}, Q_{i}$ and $R_{i, t}$ the matrices corresponding to the permutations $\left(\xi_{i} \xi_{j}\right)\left(\xi_{-i} \xi_{-j}\right),\left(\xi_{i} \xi_{-i}\right)$ and the transformations $\xi_{i}^{\prime}=t \xi_{i}, \xi_{-i}^{\prime}=t^{-1} \xi_{-i}, \quad \xi_{k}^{\prime}=\xi_{k}(k \neq i,-i)$, respectively. It is known [3] that the group $\mathfrak{D}$ is generated by the matrices $W_{i, j, t}, P_{i j}, Q_{i}$, and $R_{i, t}$. Therefore, $\mathfrak{D}^{\prime} \leqq \bar{S}$ is proved if we can show that for any distinct $|i|,|j|$, $|k|$ the matrices $\left(P_{i k} P_{i j}\right)^{2}, Q_{i} Q_{j}, R_{i, t} R_{j, t}^{-1}$, and $R_{i, t}^{2}$ are in $\bar{S}$. By elementary computations we have

$$
\begin{aligned}
\left(P_{i k} P_{i j}\right)^{2} & =W_{j,-k, 1} W_{-j, k, 1} W_{j,-k, 1} W_{i,-j, 1} W_{-i, j, 1} W_{i,-j, 1} \\
Q_{i} Q_{j} & =W_{j,-i, 1} W_{-j, i, 1} W_{j,-i, 1} W_{i, j, 1} W_{-i,-j, 1} W_{i, j 1} \\
R_{i, t} R_{j, t}^{-1} & =W_{-i, j, t-1} W_{-j, i, 1} W_{-i, j, s} W_{-j, i,-t} \\
R_{i, t} R_{j, t} & =W_{-i,-j, t-1} W_{j, i, 1} W_{-i,-j, s} W_{j, i,-t}
\end{aligned}
$$

where $s=t^{-1}-1$. Thus the matrices $\left(P_{i k} P_{i j}\right)^{2}, Q_{i} Q_{j}, R_{i, t} R_{j, t}^{-1}$ are shown to be in $\bar{\Xi}$. Since $R_{i, t}$ and $R_{j, t}$ commute, we have $R_{i, t}^{2}=\left(R_{i, t} R_{j, t}^{-1}\right)\left(R_{i, t} R_{j, t}\right)$. Hence $R_{i, t}^{2}$ is also in $\overline{\mathbb{S}}$. Therefore $\mathfrak{D}^{\prime} \leqq \bar{\Xi}$ is proved.

Now we shall show $\bar{\S} \leqq \mathfrak{O}^{\prime}$, assuming that $3 \leqq n$. For any $W_{i, j, t}$ take $k$ such that $|k| \neq|i|,|j|$. Then we have

$$
W_{i, j, t}=W_{-k, j,-1}^{-1} W_{i, k, \imath}^{-1} W_{-k, j,-1} W_{i, k, \iota}
$$

which shows that $\bar{\Im} \leqq \mathfrak{D}^{\prime}$. Therefore we have $\overline{\mathscr{S}}=\mathfrak{O}^{\prime}$ provided that $3 \leqq n$, and hence $\leftrightarrow\left(D_{n}, K\right)$ is the commutator group of projective orthogonal group defined by the quadratic form $\sum_{i=1}^{n} \xi_{i} \xi_{-i}$. 
7. The group $B\left(B_{n}, K\right)$. First we consider the case where $K$ is not of characteristic 2 . We shall show that the group $\bar{\Xi}\left(B_{n}, K\right)$ is isomorphic to the commutator group $\mathfrak{D}^{\prime}$ of the orthogonal group $\mathfrak{D}$ defined by the quadratic form $\sum_{i=0}^{n} \xi_{i} \xi_{-i}$, provided that $2 \leqq n$. By (2.4), the group $\bar{\xi}$ is generated by the matrices

$$
\begin{aligned}
W_{i, j, t} & =I+t\left(E_{i,-j}-E_{j,-i}\right), \\
V_{i, t} & =I+t\left(2 E_{-i 0}-E_{0 i}\right)-t^{2} E_{-i i},
\end{aligned}
$$

where $t \in K, i, j= \pm 1, \cdots, \pm n ; i \neq \pm \pm j$. Define the matrices $P_{i j}, Q_{i}, R_{i, t}$ as in $\S 6$ for $i, j \neq 0$. It is known [4] that the group $\mathfrak{D}$ is generated by the matrices $W_{i, j, t}, V_{i, t}, P_{i j}, Q_{i}, R_{i, t}$. The formula (6.1) shows $\mathfrak{D}^{\prime} \leqq \bar{\Xi}$.

We shall show $\bar{\Theta} \leqq \mathfrak{O}^{\prime}$ under the assumption that $2 \leqq n$. By an elementary computation we have

$$
W_{i, j, 2 \imath}=V_{i, t} V_{j, 1} V_{i, t}^{-1} V_{j, 1}^{-1},
$$

which shows that every $W_{i, j, t}$ is in $\mathfrak{D}^{\prime}$, since $K$ is not of characteristic 2 . Also we have

$$
V_{i, t}=W_{j i, t^{2}}\left(W_{-j i, 1}^{-1} V_{j,-t}^{-1} W_{-j i, 1} V_{j,-t}\right) .
$$

Hence we see that $V_{i, t}$ is also in $\mathfrak{O}^{\prime}$. Thus $\bar{\Im} \leqq \mathfrak{D}^{\prime}$, and hence $\overline{\mathscr{S}}=\mathfrak{O}^{\prime}$ is proved. Therefore if $K$ is not of characteristic 2 then $B\left(B_{n}, K\right)$ is the commutator group of the projective orthogonal group defined by the quadratic form $\sum_{i=0}^{n} \xi_{i} \xi_{-i}$.

Consider now the case where $K$ is of characteristic 2. Denote by $\mathfrak{M}$ a vector space over $K$ spanned by the indeterminates $\xi_{i}, i=0, \pm 1, \cdots, \pm n$, and by $\mathfrak{M}^{\prime}$ the subspace of $\mathfrak{M}$ spanned by $\xi_{i}, i= \pm 1, \cdots, \pm n$. The matrix $W_{i, j, t}$ is the matrix of the linear transformation of $\mathfrak{M}:$

$$
\xi_{i}^{\prime}=\xi_{i}+t \xi_{-j}, \quad \xi_{j}^{\prime}=\xi_{j}+t \xi_{-i}, \quad \xi_{k}^{\prime}=\xi_{k} \quad(k \neq i, j),
$$

while $V_{i, t}=I+t E_{0 i}+t^{2} E_{-i i}$ is the matrix of the linear transformation:

$$
\xi_{0}^{\prime}=\xi_{0}+t \xi_{i}, \quad \xi_{-i}^{\prime}=\xi_{-i}+t^{2} \xi_{i}, \quad \xi_{k}^{\prime}=\xi_{k} \quad(k \neq 0,-i) .
$$

Therefore the subspace $\mathfrak{M}^{\prime}$ reduces the group $\bar{\xi}$. Moreover, (7.1) and (7.2) shows that the linear transformations in $\bar{G}$ leave the bilinear form $f_{C}(\xi, \eta)$ $=\sum_{i=1}^{n}\left(\xi_{i} \eta_{-i}-\xi_{-i} \eta_{i}\right)$ invariant. It was noted in $\S 3$ that the center of $\bar{B}$ consists of elements of the form $a I$ with $a \in K$. Since $a I$ leaves $f_{C}(\xi, \eta)$ invariant, we have $a^{2}=1$ and hence $a=1$. Then $(B=\bar{S}$ and hence $\bar{B}$ is simple. (We exclude the exceptional cases. See $[2$, p. 65]). Therefore the representation of $\bar{S}$ induced in the space $\mathfrak{M}^{\prime}$ is faithful. Thus we may regard (S) as a multiplicative group of $2 n \times 2 n$ matrices generated by $W_{i, j, t}=I+t\left(E_{i,-j}-E_{j,-i}\right)$ and $V_{i, t}=I+t^{2} E_{-i i}$, where $t \in K$ and $i, j= \pm 1, \pm 2, \cdots, \pm n$. We have mentioned in $\$ 5$ that the symplectic group $\operatorname{Sp}(2 n, K)$ is generated by $W_{i, j, t}$ and $I+t E_{-i i}$. Thus the group $(S)\left(B_{n}, K\right)$, in the case when $K$ is of characteristic 2, 
is a subgroup of $\operatorname{Sp}(2 n, K)$. If, however, the field $K$ is perfect then $\leftrightarrow=\operatorname{Sp}(2 n$, $K)$.

8. The algebra $G_{2}$ as a subalgebra of $B_{3}$. E. Cartan $[1$, p. 146] gave a representation of $G_{2}$ as a subalgebra of the algebra $B_{3}$, which we shall derive here in a form convenient for our purposes. The algebra $B_{3}$ is defined as the algebra of all $7 \times 7$ matrices $X$ satisfying $f_{B}(X \xi, \eta)+f_{B}(\xi, X \eta)=0$ identically, where

$$
f_{B}(\xi, \eta)=2 \xi_{0} \eta_{0}+\sum_{i=1}^{3}\left(\xi_{-i} \eta_{i}+\xi_{i} \eta_{-i}\right) .
$$

Denote by $E_{i j}$ the $7 \times 7$ matrix such that $\xi^{\prime}=E_{i j} \xi$ where $\xi_{i}^{\prime}=\xi_{j}, \xi_{k}^{\prime}=0$ for $k \neq i$, and set

$$
\begin{aligned}
& F_{i j}=E_{-i j}-E_{-j i}, \quad F_{i 0}=2 E_{-i 0}-E_{0 i}, \quad F_{0 i}=-F_{i 0}, \\
& H_{i j}=F_{i,-i}+F_{j,-j}, \quad H_{i 0}=2 F_{i,-i}, \quad H_{0 i}=2 F_{i,-i}
\end{aligned}
$$

for $i, j=1,2,3$. Then $\left\{H_{r}\right\}=\left\{H_{i j} \mid i<j, i \neq-j\right\}$ is the set of all co-weights of $B_{3}$ with respect to the Cartan subalgebra $\mathfrak{h}$ spanned by all $H_{r}$, and $\left\{X_{r}\right\}$ $=\left\{F_{i j} \mid i<j, i \neq-j\right\}$ is a system of root vectors such that if $X_{r}=F_{i j}$ then $H_{r}=H_{i j}$ and $X_{-r}=F_{-j,-i}$. It was shown in [5] that there is an automorphism $\phi$ of $B_{3}$ such that $\phi\left(X_{r}\right)=-X_{-r}$ for all roots $r$ of $B_{3}$. Cartan showed that the six elements

$$
U_{i j}=F_{i,-j}, \quad(0<i, 0<j, i \neq j),
$$

together with the six elements

$$
U_{0 i}=F_{i 0}+F_{i^{\prime} i^{\prime \prime}}, \quad U_{i 0}=F_{0,-i}+F_{-i^{\prime \prime},-i^{\prime}},
$$

where $i=1,2,3$ and where $\left(i i^{\prime} i^{\prime \prime}\right)$ is a even permutation of $1,2,3$, form a system of root vectors of a subalgebra $g$ of $B_{3}$ isomorphic to $G_{2}$. The Cartan subalgebra of $\mathfrak{g}$ is given by $\mathfrak{g} \cap \mathfrak{h}$. We have

$$
\begin{aligned}
& {\left[U_{i j}, U_{j i}\right]=H_{i,-j},} \\
& {\left[U_{0 i}, U_{i 0}\right]=H_{i 0}+H_{i^{\prime} i^{\prime \prime}},}
\end{aligned}
$$

where $i \neq 0, j \neq 0$, since $\left[F_{i 0}, F_{-i^{\prime \prime},-i^{\prime}}\right]=0,\left[F_{0,-i}, F_{i^{\prime} i^{\prime \prime}}\right]=0$. We have also the identities

$$
\left[H_{i,-j}, U_{i j}\right]=2 U_{i j}, \quad\left[H_{i 0}+H_{i^{\prime} i^{\prime \prime}}, U_{0 i}\right]=2 U_{0 i} .
$$

Hence we see that the elements on the right hand side of (8.1) are co-weights of the algebra $\mathrm{g}$. Moreover, it is easily seen that the automorphism $\phi$ of $B_{3}$ induces an automorphism of the subalgebra $g$ such that $\phi\left(U_{i j}\right)=-U_{j i}$, $\phi\left(U_{0 i}\right)=-U_{i 0}$. Therefore the system $\left\{U_{i j}, U_{0 i}, U_{i 0}\right\}$ of root vectors can be used to define the group $(S)\left(G_{2}, K\right)$.

9. The group $\left(G_{2}, K\right)$. Let $K$ be an arbitrary field. By the same argument as in $\$ 3$ we see that $\$\left(G_{2}, K\right)$ is isomorphic to the quotient group over 
its center of the multiplicative group $\bar{\Im}\left(G_{2}, K\right)$ generated by the matrices

$$
\left\{\begin{array}{l}
\exp \left(t U_{i j}\right)=I+t U_{i j}, \\
\exp \left(t U_{0 i}\right)=I+t U_{0 i}-t^{2} E_{-i i}, \\
\exp \left(t U_{i 0}\right)=I+t U_{i 0}-t^{2} E_{-i i},
\end{array}\right.
$$

where $t \in K$ and $i, j=1,2,3$.

Dickson $[4, \S 9 ; 5]$, guided by the above mentioned representation of the algebra $G_{2}$ given by Cartan, considered the multiplicative group $\mathfrak{S}$ consisting of all $7 \times 7$ matrices $X$ with entries in $K$ which satisfy the conditions (9.2)(9.4):

(9.2) $\operatorname{det} X=1$;

(9.3) $X$ leaves the quadratic form

$$
q(\xi)=\xi_{0}^{2}+\xi_{1} \xi_{-1}+\xi_{2} \xi_{-2}+\xi_{3} \xi_{-3}
$$

invariant, that is, $q(X \xi)=q(\xi)$ identically;

(9.4) $X$ leaves invariant the system of equations

$$
\xi_{0} \eta_{i}-\xi_{i} \eta_{0}+\xi_{-i^{\prime}} \eta_{-i^{\prime \prime}}-\xi_{-i^{\prime \prime}} \eta_{-i^{\prime}}=0
$$

where $\left(i i^{\prime} i^{\prime \prime}\right)$ are cyclic even permutations of $1,2,3$, or of $-1,-2,-3$, when $X$ operates cogrediently upon the two sets of variables $\left(\xi_{i}\right),\left(\eta_{i}\right)$, $-3 \leqq i \leqq 3$.

Dickson proved, assuming that $K$ has more than two elements, that the group $\mathfrak{S}$ is simple and generated by the matrices in (9.1) and the matrices $R_{i, t} R_{j, t}^{-1}, i \neq j, i \neq 0, j \neq 0, t \in K$, where $R_{i, t}$ is the matrix of the transformation

$$
\xi_{i}^{\prime}=t \xi_{i}, \quad \xi_{-i}^{\prime}=t^{-1} \xi_{-i}, \quad \xi_{k}^{\prime}=\xi_{k} \quad(k \neq i,-i) .
$$

In view of the formulas (6.1), the matrix $R_{i, t} R_{j, t}^{-1}$ can be represented as a product of matrices in (9.1), and hence it follows that the group $\mathfrak{S}$ is generated by the matrices in (9.1). Therefore we have $\mathfrak{S}=\overline{\mathfrak{S}}\left(G_{2}, K\right)$. Since $\mathfrak{S}$ is simple, we have also $\mathfrak{B S}\left(G_{2}, K\right)=\overline{\mathfrak{S}}\left(G_{2}, K\right)=\mathfrak{S}$.

\section{REFERENCES}

1. E. Cartan, Thèse, Paris, 1894.

2. C. Chevalley, Sur certains groupes simples, Tôhoku Math. J. 2d ser. vol. 7 (1955) pp. 1466.

3. L. E. Dickson, The structure of certain linear groups with quadratic invariants, Proc. London Math. Soc. vol. 30 (1898-1899) pp. 70-98. 363-394.

4. - Linear groups in an arbitrary field, Trans. Amer. Math. Soc. vol. 2 (1901) pp.

5. - A new system of simple groups, Math. Ann. vol. 60 (1905) pp. 137-150.

6. - Linear groups, Leipzig, Teubner, 1901.

7. E. B. Dynkin, The structure of semi-simple algebras, Uspehi Matematiceskih Nauk (N.S.) vol. 2 no. 4 (20) (1947) pp. 59-127, Amer. Math. Soc. Translations no. 17.

University of British Columbia, VANCOUVER, B. C. 